\title{
Do tonic and burst TMS modulate the lemniscal and extralemniscal system diifierentially?
}

\author{
Dirk De Ridder 1, Elsa van der Loo 1, Karolien Van der Kelen 1, Tomas Menovsky 1, Paul van de Heyning 1, \\ Aage Moller 2
}

1. Dept of Neurosurgery and ENT, University Hospital Antwerp, Belgium

2. School of Behavioral and Brain Science, University of Texas at Dallas, Dallas, USA

Correspondence to: Dirk De Ridder, Dept of Neurosurgery, University Hospital Antwerp, Wilrijkstraat 10, 2650 Edegem, Belgium. Tel: +32 3 8213336; Fax: +32 3 8252428; dirk.de.ridder@neurosurgery.be

Received: 2007.06.22; Accepted: 2007.10.08; Published: 2007.10.09

Introduction: Tinnitus is an auditory phantom percept related to tonic and burst hyperactivity of the auditory system. Two parallel pathways supply auditory information to the cerebral cortex: the tonotopically organised lemniscal system, and the non-tonotopic extralemniscal system, firing in tonic mode and burst mode respectively. Transcranial magnetic stimulation (TMS) is a non-invasive method capable of modulating activity of the human cortex, by delivering tonic or burst stimuli. Burst stimulation is shown to be more powerful in activating the cerebral cortex than tonic stimulation and bursts may activate neurons that are not activated by tonic stimulations.

Methods: The effect of both tonic and burst TMS in 14 placebo-negative patients presenting narrow band/white noise tinnitus were analysed.

Results: Our TMS results show that narrow band/white noise tinnitus is better suppressed with burst TMS in comparison to tonic TMS, $t(13)=6.4, p=.000$. For pure tone tinnitus no difference is found between burst or tonic $\mathrm{TMS}, t(13)=.3$, ns.

Discussion: Based on the hypothesis that white noise is the result of hyperactivity in the non-tonotopic system and pure tone tinnitus of the tonotopic system, we suggest that burst stimulation modulates the extralemniscal system and lemniscal system and tonic stimulation only the lemniscal system.

Key words: Burst, extralemniscal, lemniscal, TMS, Tonic

\section{Introduction}

Tinnitus is an auditory phantom percept [1, 2] related to reorganization [2] and hyperactivity [3] of the auditory system. The auditory system consists of two main parallel pathways supplying auditory information to the cerebral cortex: the tonotopically organized lemniscal (classical) system, and the non-tonotopic extralemniscal (non-classical) system. The classical pathways use the ventral thalamus, the neurons of which project to the primary auditory cortex whereas the non-classical pathways use the medial and dorsal thalamic nuclei that project to the secondary auditory cortex and association cortices, thus bypassing the primary cortex [4]. While neurons in the classical pathways only respond to one modality of sensory stimulation, many neurons in the non-classical pathway respond to more than one modality. Neurons in the ventral thalamus fire in a tonic or semi-tonic mode while neurons in the medial and dorsal thalamus fire in bursts $[5,6]$. The non-classical pathways receive their input from the classical pathways, which means that the ascending auditory pathways are a complex system of at least two main parallel systems that provide different kinds of processing and which interact with each other in a complex way. Both systems provide sensory input to the amygdala through a long cortical route, and in addition, the non-classical pathways provide subcortical connections to the lateral nucleus of the amygdala from dorsal thalamic nuclei [7].

Studies in humans have indicated that some patients with tinnitus have an abnormal activation of the non-classical auditory system [8]. Studies of animal models of tinnitus have shown that burst firing is increased in the non-classical system [9-11] and tonic firing activity is increased in the classical system [12-17]. Interestingly, not only tonic firing but also burst firing is increased in neurons in the primary auditory cortex in animal models of tinnitus [18]. Studies in patients with intractable tinnitus have shown that tonic electrical stimuli of the primary and secondary auditory cortex can suppress pure tone tinnitus, but not white noise/narrow band noise tinnitus [19].

We tested the hypothesis that white noise tinnitus may be caused by increased burst firing in the non-tonotopic (extralemniscal) system, whereas pure tone tinnitus may be the result of increased tonic firing 
in the tonotopic (lemniscal) system. Transcranial magnetic stimulation (TMS) is a non-invasive tool by means of which neural structures of the brain can be stimulated by the induced electrical current. It has been shown that TMS of the auditory cortex can modulate the perception of tinnitus in some patients [20-24]. TMS machines can deliver both tonic and burst stimuli (figure 1), and it has been demonstrated that tonic stimulation can suppress pure tone tinnitus, but not narrow band noise, whereas burst TMS can suppress narrow band or white noise tinnitus (De Ridder et al., submitted).

We used tonic and burst TMS aimed at the auditory cortex, to suppress unilateral pure tone and narrow band/white noise tinnitus respectively. The purpose was to elucidate the neural mechanisms of tinnitus and to develop a diagnostic tool that could distinguish between different types of tinnitus that may benefit from different kinds of treatment.

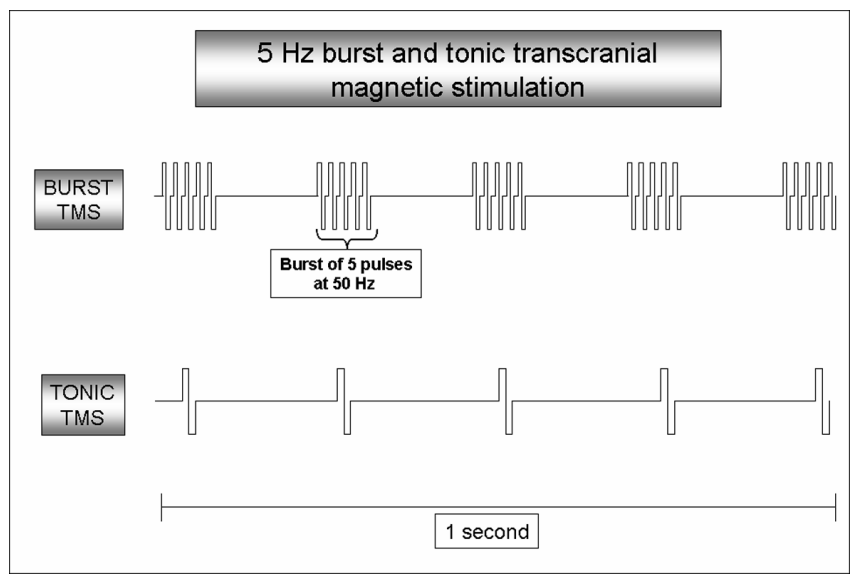

Figure 1: Five $\mathrm{Hz}$ burst and tonic TMS: $5 \mathrm{~Hz}$ burst TMS consists of 5 bursts per second, each burst consisting of 5 rapid TMS pulses eg at $50 \mathrm{~Hz}$. Five $\mathrm{Hz}$ tonic TMS consists of 5 tonic pulses per second.

\section{Methods}

We studied the effect of TMS in 70 individuals with unilateral tinnitus and compared the effect of tonic and burst stimulation of the auditory cortex evaluating the effect of such stimulation on the patients' tinnitus. The presence of a placebo effect is tested by placing the coil perpendicular to the auditory cortex at the frequencies that yield maximal tinnitus suppression rates both for tonic and burst TMS. Of the participants presenting with pure tone tinnitus, only 14 had no placebo effect on both tonic and burst TMS. Only results from these 14 patients were analyzed (7 women, 7 men; mean age 56.2 years; range 46-70 years). Of the participants presenting with narrow band/white noise tinnitus, also only 14 patients had no placebo effect on both tonic and burst TMS (7 women, 7 men; mean age 51.6 years; range 40-72 years). Results from these 28 patients, representing two comparable homogenous groups, were analyzed. Since the TMS machine generates a clicking sound on each magnetic pulse delivery, using only results from placebo negative patients prevents the possible influence of sound from the TMS masking the tinnitus. The TMS is done as a part of a continuing clinical protocol for selection of candidates for implantation of permanent electrodes for electrical stimulation of the auditory cortex for treatment for tinnitus $[19,25]$ at the multidisciplinary tinnitus clinic of the University Hospital of Antwerp, Belgium. All prospective participants undergo a complete audiological, ENT and neurological investigation to rule out possible treatable causes for their tinnitus. Tinnitus matching is performed by presenting sounds to the ear in which the tinnitus is not perceived, and both tinnitus pitch and tinnitus intensity (above hearing threshold) are matched to the perceived tinnitus. Technical investigations include MRI of the brain and posterior fossa, pure tone and speech audiometry, Auditory Brainstem Response (ABR) and tympanometry. Assessment of the tinnitus severity is analysed by Visual Analogue Scale (VAS) and Tinnitus Questionnaire[26] (TQ). Tinnitus duration is also recorded. This study is approved by the ethical committee of the University Hospital Antwerp, Belgium.

TMS is performed using a super rapid stimulator (Magstim Inc, Wales, UK) with the figure of eight coil placed over the auditory cortex contralateral to the tinnitus side, in a way previously described [21].

Before the TMS session, patients grade their tinnitus on a VAS. The motor threshold to TMS is first determined by placing the coil over the motor cortex. With the first and second digit opposed in a relaxed position, the intensity of the magnetic stimulation is slowly increased until a clear contraction is observed in the contralateral thenar muscle.

Since TMS has a poor spatial resolution, and it has been shown that results for tinnitus suppression with and without neuronavigation are not significantly different [27], the auditory cortex is targeted in this study using external landmarks: the auditory cortex is located 5-6 cm cranially to the entrance of external auditory meatus in a straight line to the vertex. After the motor threshold is determined the coil is moved to a location over the auditory cortex contralateral to the side to where the patients refer their tinnitus.

With the intensity of the stimulation set at $90 \%$ of the motor threshold, the site of maximal tinnitus suppression is determined using $1 \mathrm{~Hz}$ stimulation. During the stimulation, the patient is asked to estimate the decrease in tinnitus in percentage using the VAS. The procedure is repeated with stimulations at $5 \mathrm{~Hz}$, $10 \mathrm{~Hz}$ and $20 \mathrm{~Hz}$, each stimulation session consisting of 200 pulses. Burst stimulation is performed in a similar fashion. Bursts are presented at 5, 10 and $20 \mathrm{~Hz}$ (theta, alpha and beta burst stimulation with 3, 5, 10 pulses in each burst respectively).

\section{Statistical analysis}

Data were analysed with SPSS 13.0. Tinnitus suppression (\% reduction of tinnitus perception) data 
were analysed using a GLM with repeated measures with TMS stimulation (Tonic vs. Burst) as within-participant variable, tinnitus type (white noise vs. pure tone) as between subject factor. Differences of TMS burst or tonic stimulation on white noise tinnitus on the one hand and pure tone tinnitus on the other where explored using a paired sampled $t$-test with TMS stimulation as dependent variable and tinnitus type as grouping factor. To assess differences between genders in burst and tonic TMS stimulation, independent sampled $t$-tests were performed for white noise and pure tone tinnitus, with burst and tonic TMS stimulation as dependent variables and gender as grouping variable. To assess differences in distress caused by tinnitus depending on the side (left or right) an independent sampled t-test was performed with Tinnitus Questionnaire (TQ) score as dependent variable and tinnitus side as grouping variable. Pearson's correlations were performed to assess significant correlations between variables.

\section{Results}

The data reveal a significant main effect of TMS stimulation (Tonic vs. Burst), where burst TMS elicits significant better tinnitus suppression in general $(M=55.5 \%$, SEM $=6.0)$ than tonic TMS $(M=35.2 \%$, SEM=5.7, $\quad F(1,26)=8.9, \quad p<.01)$. Furthermore, a significant main effect of tinnitus type (white noise $v s$. pure tone) is found, with better effects for patients suffering from pure tone tinnitus $(M=55.9 \%$, SEM=6.8), than for patients suffering from white noise tinnitus $(M=34.8 \%, \mathrm{SEM}=6.8, F(1,26)=4.8, p<.05)$. In addition data reveal an interaction effect between TMS stimulation and tinnitus type $F(1,26)=12.7, p<.001$. Further paired-sampled $t$-tests show that white noise tinnitus is better suppressed with burst TMS in comparison to tonic TMS, $t(13)=6.4, p<.000$ (Figure 2). For pure tone tinnitus no difference is found between burst or tonic TMS, $t(13)=.3$, ns. No significant differences in tinnitus suppression is found between genders nor for burst TMS, $t(26)=.74, n s$., nor for tonic TMS, $t(26)=.32$, ns. Left sided tinnitus (pure tone and white noise) is perceived as more distressing than right sided tinnitus, $t(20)=1.07, p<.05$.

Some other significant correlations are noted. The longer the tinnitus exists the poorer the tinnitus suppression with tonic TMS $(r=-0.4, p<0.05)$. The TMS frequency that maximally suppresses pure tone tinnitus via tonic TMS is always the same as the burst TMS that maximally suppresses the pure tone tinnitus $(r=1, p<0.000)$, which is not so in white noise tinnitus $(r=-.4, n s$.$) .$

Figure 2: Mean tinnitus suppression (\%) for white noise and pure tone tinnitus with tonic and burst TMS stimulation

\section{Discussion}

The mechanisms of action of rTMS in tinnitus remain unclear [28].It is known that rTMS can only modulate superficial cortical areas directly. However, the primary auditory cortex which is located on Heschl's gyrus [29] is lying embedded in the posterior part of the sylvian sulcus and it is doubtful that electromagnetic fields generated by rTMS reach the primary auditory cortex when rTMS is applied over the temporal cortex. On the other hand it has been demonstrated that rTMS has effects on sites in remote structures functionally connected with the stimulated region [30]. rTMS probably modulates corticofugal pathways, as it has been shown that auditory cortex rTMS induces thalamic changes in grey matter density [31]. This is in accordance with electrical stimulation data that have shown an alteration in outer hair cell function as measured by otoacoustic emissions [32]. As there exist two corticofugal pathways from the auditory cortex [33, 34], with a different chemoarchitectonic structure and different firing patterns it is conceivable that burst and tonic rTMS modulates these pathways differentially.

The findings suggest that tonic TMS only modulates neural activity in the classical auditory system and burst TMS acts on the non-classical system directly. The results from TMS in tinnitus patients confirm the hypothesis that burst stimulation only modifies the extralemniscal system.

This suggests that hyperactivation of this non-tonotopic part of the auditory system could lead to white noise, which cannot be suppressed by tonic stimulation but only by burst stimulation, being a more powerful stimulus to modulate the cortex.

The fact that white noise can only be suppressed by burst TMS, but that burst TMS can suppress both pure tone tinnitus, suggests that burst stimulation can modulate the extralemniscal and lemniscal system, whereas tonic stimulation can only modulate the lemniscal system thus supporting the hypothesis that the non-classical system provides input to the lemniscal system $[35,36]$.

The burst TMS that maximally suppresses pure tone tinnitus TMS is the same frequency that maximally suppresses pure tone tinnitus via tonic TMS, suggesting that the extralemniscal system drives

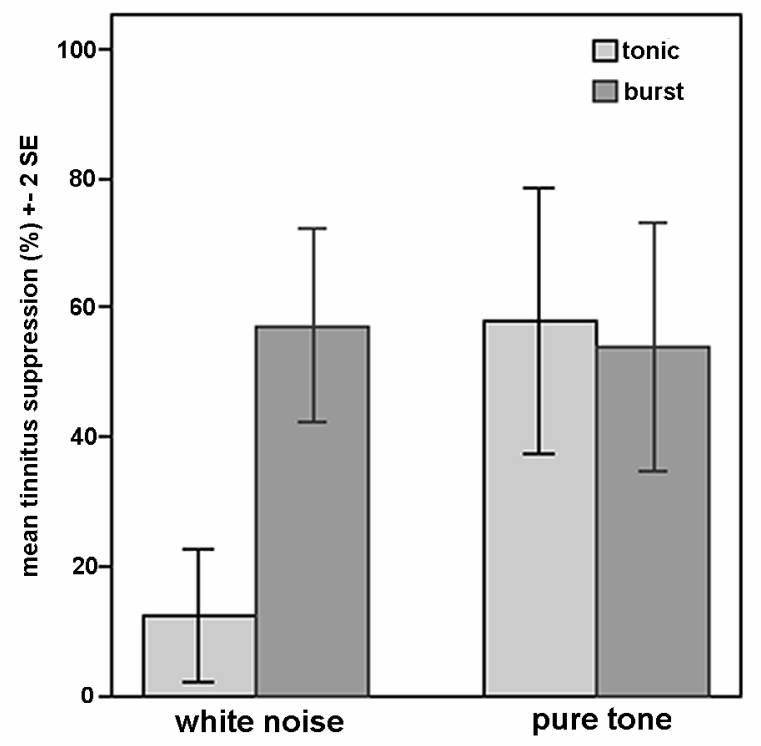


the lemniscal system as has been suggested $[35,36]$. In white noise, supposedly generated in the extralemniscal system, this is not seen, a further argument along the same line.

We have previously shown (submitted, De Ridder et al.) that lower frequencies of narrow band tinnitus respond better to burst stimulation than higher frequencies. This could be viewed as supportive of the hypothesis as well, as it is known that lower pitch sounds have a wider tuning curve and thus respond more like a non-tonotopic system in general.

Our findings also demonstrate that the longer the tinnitus exists the poorer the tinnitus can be suppressed using tonic TMS. This is in accordance with a previous study on other patients from the same institute [21].

In this study left sided tinnitus is perceived as more distressing than right sided tinnitus. This is in accordance with published epidemiological data that show that tinnitus seems to be more predominant on the left [37] and that people suffering left sided tinnitus complain more from tinnitus than people with right sided tinnitus [38].

A recent multicenter review paper on rTMS in tinnitus concluded that 'rTMS is a promising technique in the management of chronic, subjective tinnitus' ... 'However, there are still important questions to address before considering rTMS as a realistic treatment for tinnitus.' And indeed rTMS is still largely a research tool, as is stated in the rest of the conclusion of the same paper: 'Both basic research and multicentre clinical studies with large number of patients and long-term follow-up are necessary to delineate the place of rTMS in this domain.' Whereas rTMS doesn't seem to be a clinically applicable treatment for tinnitus it can potentially benefit pathophysiological studies such as these. rTMS can possibly help to select surgical candidates for permanent implants as also mentioned in this review paper. 'The fast development of implanted procedures of cortical stimulation, already initiated in tinnitus treatment, will be probably the most serious challenge to future therapeutic application of rTMS. Nevertheless, rTMS might serve at least as an important predictive test before implantation' [28].

A more interesting potential prospect of this study is that all sensory systems, the limbic system and the motor system are built in a similar way, consisting of a topographic and non-topographic pathway functioning in parallel. The data presented here suggest it could be worthwhile to verify the differential effect of tonic and burst stimulation in other pathologies of the sensory, limbic and motor systems.

\section{Conflict of interest}

The authors have declared that no conflict of interest exists.

\section{References}

1. Jastreboff PJ. Phantom auditory perception (tinnitus): mechanisms of generation and perception. Neurosci Res
1990;8(4):221-54.

2. Muhlnickel W, Elbert T, Taub E, Flor H. Reorganization of auditory cortex in tinnitus. Proc Natl Acad Sci U S A 1998;95(17):10340-3.

3. Eggermont JJ, Roberts LE. The neuroscience of tinnitus. Trends Neurosci 2004;27(11):676-82.

4. Møller AR. Sensory Systems: Anatomy and Physiology. Amsterdam: Academic Press, 2003.

5. He J, Hu B. Differential distribution of burst and single-spike responses in auditory thalamus. J Neurophysiol 2002;88(4):2152-6.

6. Hu B, Senatorov V, Mooney D. Lemniscal and non-lemniscal synaptic transmission in rat auditory thalamus. J Physiol 1994;479 ( Pt 2):217-31.

7. LeDoux JE. Emotional memory systems in the brain. Behav Brain Res 1993;58(1-2):69-79.

8. Moller AR, Moller MB, Yokota M. Some forms of tinnitus may involve the extralemniscal auditory pathway. Laryngoscope 1992;102(10):1165-71.

9. Chen GD, Jastreboff PJ. Salicylate-induced abnormal activity in the inferior colliculus of rats. Hear Res 1995;82(2):158-78.

10. Eggermont JJ, Kenmochi M. Salicylate and quinine selectively increase spontaneous firing rates in secondary auditory cortex. Hear Res 1998;117(1-2):149-60.

11. Eggermont JJ. Central tinnitus. Auris Nasus Larynx 2003;30: S7-12.

12. Brozoski TJ, Bauer CA, Caspary DM. Elevated fusiform cell activity in the dorsal cochlear nucleus of chinchillas with psychophysical evidence of tinnitus. J Neurosci 2002;22(6):2383-90.

13. Zhang JS, Kaltenbach JA. Increases in spontaneous activity in the dorsal cochlear nucleus of the rat following exposure to high-intensity sound. Neurosci Lett 1998;250(3):197-200.

14. Zacharek MA, Kaltenbach JA, Mathog TA, Zhang J. Effects of cochlear ablation on noise induced hyperactivity in the hamster dorsal cochlear nucleus: implications for the origin of noise induced tinnitus. Hear Res 2002;172(1-2):137-43.

15. Kaltenbach JA, Afman CE. Hyperactivity in the dorsal cochlear nucleus after intense sound exposure and its resemblance to tone-evoked activity: a physiological model for tinnitus. Hear Res 2000;140(1-2):165-72.

16. Kaltenbach JA, Godfrey DA, Neumann JB, McCaslin DL, Afman $\mathrm{CE}$, Zhang J. Changes in spontaneous neural activity in the dorsal cochlear nucleus following exposure to intense sound: relation to threshold shift. Hear Res 1998;124(1-2):78-84.

17. Kaltenbach JA, Zacharek MA, Zhang J, Frederick S. Activity in the dorsal cochlear nucleus of hamsters previously tested for tinnitus following intense tone exposure. Neurosci Lett 2004;355(1-2):121-5.

18. Ochi K, Eggermont JJ. Effects of quinine on neural activity in cat primary auditory cortex. Hear Res 1997;105(1-2):105-18.

19. De Ridder D, De Mulder G, Verstraeten E, et al. Primary and secondary auditory cortex stimulation for intractable tinnitus. ORL 2006; in press.

20. Plewnia C, Bartels M, Gerloff C. Transient suppression of tinnitus by transcranial magnetic stimulation. Ann Neurol 2003;53(2):263-6.

21. De Ridder D, Verstraeten E, Van der Kelen K, De Mulder G, Sunaert S, Verlooy J, Van de Heyning P, Moller A. Transcranial magnetic stimulation for tinnitus : influence of tinnitus duration on stimulation parameter choice and maximal tinnitus suppression. Otol Neurotol 2005;26(4):616-9.

22. Eichhammer P, Langguth B, Marienhagen J, Kleinjung T, Hajak G. Neuronavigated repetitive transcranial magnetic stimulation in patients with tinnitus: a short case series. Biol Psychiatry 2003;54(8):862-5.

23. Kleinjung T, Eichhammer P, Langguth B, Jacob P, Marienhagen 
J, Hajak G, Wolf SR, Strutz J. Long-term effects of repetitive transcranial magnetic stimulation (rTMS) in patients with chronic tinnitus. Otolaryngol Head Neck Surg 2005;132(4):566-9.

24. Londero A, Lefaucheur JP, Malinvaud D, Brugieres P, Peignard P, Nguyen JP, Avan P, Bonfils P. [Magnetic stimulation of the auditory cortex for disabling tinnitus: preliminary results]. Presse Med 2006;35(2 Pt 1):200-6.

25. De Ridder D, De Mulder G, Walsh V, Muggleton N, Sunaert S, Moller A. Magnetic and electrical stimulation of the auditory cortex for intractable tinnitus. Case report. J Neurosurg 2004;100(3):560-4.

26. Goebel G, Hiller W. [The tinnitus questionnaire. A standard instrument for grading the degree of tinnitus. Results of a multicenter study with the tinnitus questionnaire]. Hno 1994;42(3):166-72.

27. Langguth B, Zowe M, Landgrebe M, Sand $P$, Kleinjung T, Binder $H$, Hajak G, Eichhammer P. Transcranial magnetic stimulation for the treatment of tinnitus: a new coil positioning method and first results. Brain Topogr 2006;18(4):241-7.

28. Londero A, Langguth B, De Ridder D, Bonfils P, Lefaucheur JP. Repetitive transcranial magnetic stimulation (rTMS): a new therapeutic approach in subjective tinnitus? Neurophysiol Clin 2006;36(3):145-55.

29. Clarke S, Rivier F. Compartments within human primary auditory cortex: evidence from cytochrome oxidase and acetylcholinesterase staining. Eur J Neurosci 1998;10(2):741-5.

30. Kimbrell TA, Dunn RT, George MS, et al. Left prefrontal-repetitive transcranial magnetic stimulation (rTMS) and regional cerebral glucose metabolism in normal volunteers. Psychiatry Res 2002;115(3):101-13.

31. May A, Hajak G, Ganssbauer S, Steffens T, Langguth B, Kleinjung $\mathrm{T}$, Eichhammer $\mathrm{P}$. Structural brain alterations following 5 days of intervention: dynamic aspects of neuroplasticity. Cereb Cortex 2007;17(1):205-10.

32. Perrot $X$, Ryvlin $P$, Isnard J, Guenot $M$, Catenoix H, Fischer $C$, Mauguiere F, Collet L. Evidence for corticofugal modulation of peripheral auditory activity in humans. Cereb Cortex 2006;16(7):941-8.

33. Hazama M, Kimura A, Donishi T, Sakoda T, Tamai Y. Topography of corticothalamic projections from the auditory cortex of the rat. Neuroscience 2004;124(3):655-67.

34. Winer JA, Diehl JJ, Larue DT. Projections of auditory cortex to the medial geniculate body of the cat. J Comp Neurol 2001;430(1):27-55.

35. Jones EG. The thalamic matrix and thalamocortical synchrony. Trends Neurosci 2001;24(10):595-601.

36. Jones EG. A new view of specific and nonspecific thalamocortical connections. Adv Neurol 1998;77:49-71.

37. Axelsson A, Ringdahl A. Tinnitus--a study of its prevalence and characteristics. Br J Audiol 1989;23(1):53-62.

38. Hallberg LR, Erlandsson SI. Tinnitus characteristics in tinnitus complainers and noncomplainers. Br J Audiol 1993;27(1):19-27. 